\title{
ANÁLISIS DIFERENCIAL RETROSPECTIVO DE LAS VARIABLES DE SALUD MENTAL EN LESBIANAS, GAIS Y BISEXUALES (LGB) VÍCTIMAS DE BULLYING HOMOFÓBICO EN LA ESCUELA
}

\author{
AITOR MARTXUETA Y JUAN ETXEBERRIA \\ Faculta de Filosofía y Ciencias de la Educación, Universidad del País Vasco, San Sebastián, España
}

\begin{abstract}
Resumen: Uno de los contextos donde las personas con orientaciones afectivo-sexuales no normativas sufren mayor discriminación, violencia y rechazo es el escolar Evidencias empíricas verifican que este acoso sufrido en el pasado puede dejar una impor tante huella en el bienestar psicológico de estos sujetos. El estudio actual tuv o como principal objetivo analizar si aquellas personas lesbianas, gais y bisexuales (LGB) que sufrieron un evento estresante en la infancia y/o adolescencia, tal como haber sido víctima de acoso escolar por moti vo de orientación afectivo-sexual, tiene consecuencias negativas en su bienestar psicológico. Los resultados obtenidos, realizado con 119 sujetos LGB sugieren que el bullying sufrido en la infancia y/o adolescencia influye en el bienestar psicológico de los sujetos en la actualidad, concretamente, en los niveles de depresión y ansiedad, autoestima y balanza de afectos. Junto a esto, las dimensiones de instrumentalidad y expresividad de la identidad de género son también factores que pueden influir en el bienestar psicológico.
\end{abstract}

Palabras clave: Acoso homofóbico; bienestar psicológico; identidad de género; LGB.

Retrospective differential analysis of mental health variables in lesbians, gays and bisexuals (LGB) victims of homophobic bullying at school

\begin{abstract}
One of the contexts where persons with non-normative affective-sexual orientation suffer more discrimination, violence and rejection, is the school context. Empirical evidence verifies that this harassment, suffered in the past, may leave an important imprint on the psychological well-being of these subjects. The present study had as its first objective to analyze if lesbians, gays and bisexuals (LGB) who suffered a stressful event in childhood and/or adolescence, such as ha ving been the victim of school harassment on account of afective-sexual orientation, show negative consequences in their psychological well-being. The results, obtained from 119 LGB subjects, suggest that bullying suffered in childhood and/or adolescence af fects the subjects' psychological well-being of today and, specifically, depression and anxiety le vels, self-esteem and affective balance. Along with these factors, the instrumentality and expressiveness dimensions of gender identity can also be factors which may influence psychological well-being.
\end{abstract}

Keywords: Gender identity; homophobic bullying; LGB; psychological well-being.

\section{INTRODUCCIÓN}

Son diferentes las investigaciones que han demostrado la influencia de las experiencias homofóbicas percibidas en el impacto negativo en la salud mental de los sujetos LGB (Mireshghi y Matsumoto, 2008). En los contextos de

Recibido: 30 mayo 2013; aceptado: 2 diciembre 2013

Correspondencia: Aitor Martxueta, Departamento de Métodos de Investigación y Diagnóstico en Educación, Universidad del País Vasco, Avda. Tolosa 70, 20018 Donostia-San Sebastián. Correo-e: aitor.martxueta@ehu.es. desarrollo, el estigma social adjuntado a la no heterosexualidad y la heteronor matividad, heterosexualidad obligatoria o heteronorma (demandas de las normas de la cultura dominante), son factores a los que los sujetos LGB tienen que hacer frente. En general, este afrontamiento puede comenzar en la adolescencia, periodo en el que los sujetos LGB son más vulnerabes.

La adolescencia es un período para el desarrollo de la pubertad durante el cual aumentan las conductas sexuales y las conductas de rol de género (Eccles y Bryan, 1994) y se cuestiona el 
lugar de cada uno en el espectro de la sexualidad (Williams, Connolly, Pepler y Craig, 2005). Cuando el aspecto se xual emerge se convierte en algo más central de la identidad, hay un aumento de deseos y atracciones asociadas al desarrollo de la pubertad y es entonces cuando en jóvenes que cuestionan su se xualidad surgen preguntas con relación a sus deseos afecti vosexuales (Pilkington y D'Augelli, 1995). El contexto en el que esto ocurre, descrito por Patterson (1995) como prejuicioso y discriminatorio y regido por una orientación afecti vo-sexual predominante, el heterosexual, inhibe a los jóvenes en la exploración y desarrollo de sus identidades y deben soportar las reacciones negativas que pueden provocar en ellos. Es en este contexto, en el que predomina la heteronor ma, donde los jóvenes pueden ser percibidos como diferentes, dando lugar a las bases de acoso y victimización dentro de un entomo de iguales (Kosciw, Greytak, Diaz y Bar tkiewicz, 2010; Rivers y Noret, 2008). La estigmatización que sufren por ser o ser percibido como LGB, tiene consecuencias sociales tales como el rechazo, la $f$ alta de aceptación, la falta de integración social y la falta de apoyo social (Graber y Archibald, 2001) que pueden causar mayores dificultades en el ajuste de conductas y un riesgo ele vado de angustia emocional que incluye trastornos tales como la depresión y la ansiedad, el abuso del alcohol y drogas e incluso el suicidio (P oteat, Aragon, Espelage y Koenig, 2009; Ryan, Huebner, Diaz y Sanchez, 2009). En lo que respecta a la heteronormatividad o heterosexualidad obligatoria, mientras muchos jóvenes heterosexuales siguen el camino prescrito de las demandas, de las normas de la cultura dominante, la heteronormatividad, los jóvenes LGB tienen que afrontar la falta de estructuras sociales, modelos de comprensión, modelos de rol positivos, guiones afectivo-sexuales y orientaciones prácticas que les conduzcan a la etapa adulta. En este proceso, la desesperación, la denegación de sus opciones afectivo-sexuales y el desajuste entre un self homosexual y las expectativas de una sociedad regida por la heteronorma puede crear un estrés particular, que se genera por ser miembro de una minoría sexual.

A este respecto, el exceso de estrés que sufren aquellas personas de una cate goría social estigmatizada debido a su posición social, a menudo minoritaria, se le denomina estrés de minorías. En otros términos, el estrés de minorías hace referencia a las fuentes de estrés a las cuales están sometidas aquellas personas que pertenecen a una minoría social, a los conflictos del estatus de minoría con otros roles que sur gen como resultado del prejuicio social, de la discriminación, de la estigmatización y del estatus de minoría culturalmente sancionado, y son provocadoras de un ambiente social hostil, estresante, que genera problemas en la salud mental (Meyer, 1995, 2003, 2007).

En el caso de aquellos sujetos que pertenecen a una cate goría social estigmatizada relacionada con la sexualidad, el estrés de minorías, hace referencia, según el propio Meyer (2003), a la disonancia existente entre la heteronormatividad o heterosexualidad obligatoria y la afiliación al grupo minoritario o auto-identidad internalizada que incluye experiencias de prejuicio (experiencias de discriminación, violencia), expectativas de rechazo, esconder y ocultar sus opciones afectivo-sexuales y la homonegatividad internalizada. Diferentes estudios confirman que las condiciones sociales discriminatorias tienen efectos adversos en la salud de las personas LGB (Meyer, 2003, 2007; Takács, 2006).

Uno de los contextos donde más experiencias de discriminación y violencia sufren las personas LGB es el contexto escolar. Los estudios sobre la victimización con muestras de jóvenes LGB basadas en la orientación afectivosexual de los participantes se han relacionado con indicadores de ajuste (Bontempo y D’Augelli, 2002; Pilkington y D'Augelli, 1995). Además, las experiencias de bullying sufridas en el contexto escolar se asocian con depresión, ansiedad, baja autoestima, síntomas de estrés postraumático, abuso de sustancias, aislamiento, tentativa de suicidio y suicidio (Birkett, Espelage y Koenig, 2009; Espelage, Aragon y Birkett, 2008; FELGTB, 2012; Ferreira, Granero, Noorian, Romero y DomènechLlaberia, 2012; Magaz, Chorot, Sandin, Santed y Valiente, 2011; Quiceno, Mateus, Cardenas, Villareal y Vinaccia, 2013; Swearer, Turner, Givens y Pollack, 2008). 
Asimismo, los estudios centrados en la conducta de bullying en la escuela se han valido de los recuerdos subjetivos de los sujetos que fueron acosados y de sus acosadores (Rivers, 2004; Rivers y Cowie, 2006) con el objetivo de conocer si el estatus de víctima de aquellas personas que sufrieron bullying en la escuela permanece a lo largo de los años. Los resultados de diversas investigaciones apuntan a que el estatus de víctima parece ser relativamente estable durante el tiempo (Olweus, 1993; Rivers, 2004; Rivers y Cowie, 2006) y que algunas de estas personas continúan teniendo síntomas de estrés postraumático (trastornos psicológicos) como consecuencia de recordar las e xperiencias de acoso sufridas en la escuela (Ri vers, 2004; Rivers y Cowie, 2006). A este respecto, el bullying que las personas LGB sufren en el contexto escolar por motivo de orientación afectivo-sexual puede ser una fuente de estrés que influye en la salud mental de los mismos.

Entre los predictores del bienestar psicológico han destacado las características de instrumentalidad y expresividad relativas a la identidad de género masculina y femenina, respectivamente. Así, la instrumentalidad está relacionada con las medidas tradicionales de bienestar (Payne, 1987; Sharpe, Heppner y Dixon, 1995). Diversos estudios han mostrado el efecto moderador de la instr umentalidad en la relación entre sucesos estresantes ne gativos y síntomas psicológicos, tanto en varones como en mujeres (Barret y White, 2002; Bromberger y Matthews, 1996; Wagner y Compas, 1990; Whitley, 1984), mientras que la alta expresividad posee el efecto contrario (Wagner y Compas, 1990).

Por su parte, aunque existen pocas evidencias empíricas que hayan estudiado la relación entre el rol de género, la orientación afecti vosexual y el distrés psicológico en personas LGB, los resultados indican que las puntuaciones altas en masculinidad predijeron los índices más bajos de la depresión y las puntuaciones más altas en feminidad los índices más altos, tanto en mujeres lesbianas como en hombres gais (Carlson y Baxter, 1984). Sin embargo, los resultados parecen ser contradictorios ya que en el estudio de Blashill y Hughes (2009), la masculinidad no fue asociada de forma signifi- cativa con el distrés psicológico, ni de for ma positiva ni de forma negativa. En lo que respecta a la autoestima, las puntuaciones altas en masculinidad predijeron los índices más altos de autoestima pero solo en el caso de los hombres gais (Carlson y Baxter, 1984).

Desde este marco teórico se hipotetiza que, debido al mayor estrés social al que están e xpuestas las personas con orientaciones afectivosexuales no normativas, la prevalencia de los trastornos mentales será mayor. También se hipotetiza que aquellos sujetos que sufrieron un evento estresante en la infancia y/o adolescencia, tal como haber sido víctima de acoso en la escuela por motivo de orientación afectivo-sexual, tendrán consecuencias ne gativas en su bienestar psicológico. Para el análisis de estas hipótesis globales se ha diseñado un estudio empírico, exploratorio, descriptivo y comparativo cuyo objetivo general es evaluar en qué medida las variables objeto de estudio, las denominadas variables de salud mental (autoestima, ansiedad, depresión y balanza de afectos) son alteradas por un evento estresante vivido en el pasado, en concreto, haber sido víctima de acoso por insultos relacionados con la orientación afectivo-sexual y por los rasgos de personalidad instrumentales y/o expresivos (identidad de género).

\section{MÉTODO}

\section{Participantes}

En este estudio par ticiparon 119 sujetos LGB, 85 varones y 32 mujeres ( 2 sujetos no indicaron su sexo) con edades comprendidas entre los 17 y 57 años $(M=37.9, D T=8.24)$. El 96.6\% de los sujetos se declararon homosexuales, siendo únicamente 4 sujetos bisexuales.

El perfil sociodemográfico de la muestra nos indica que, en relación al estado civil, la mayoría de los sujetos $(75.6 \%)$ están solteros, un $14.3 \%$ tiene pareja de hecho, un $4.3 \%$ está separado o divorciado y el $3.4 \%$ vi ve en pareja legalmente inscrita. Del total de sujetos, el $56.8 \%$ tiene actualmente pareja y un $34.5 \%$ reside con ella. En lo que respecta al ni vel de 
estudios, el $47.1 \%$ posee estudios universitarios completos, el $10.9 \%$ estudios universitarios sin completar, el $26.9 \%$ tiene estudios secundarios, un $14.3 \%$ estudios primarios y tan sólo un sujeto tiene sin concluir sus estudios primarios. En lo que respecta a su ocupación, el $72.3 \%$ tiene un contrato laboral permanente, el 13.4\% tiene un trabajo temporal, un 3.4\% está jubilado o es pensionista, un 5\% son estudiantes, el 1.7\% está desempleado y únicamente una persona se dedica a las labores domésticas. En cuanto a las creencias religiosas, el $40.3 \%$ se declara católico, un $1.7 \%$ protestante, el $0.8 \%$ judío, un $1.7 \%$ indica pertenecer a otra religión, el $27.7 \%$ declara no tener religión y un $27.7 \%$ no cree en dios. Por su parte, el $63.9 \%$ indica que no practica la religión, el $24.3 \%$ lo hace ocasionalmente y un $7.6 \%$ lo hace a menudo o todos los días. En cuanto a la ideolo gía política, el $25 \%$ se posiciona en la extrema izquierda, un $60.3 \%$ se autodefine como de izquierda moderada, el $11.2 \%$ se considera de centro y el $3.5 \%$ manifiesta una alta simpatía por la derecha moderada. Por último, y en lo que respecta al nivel de ingresos económicos mensuales, el $26.1 \%$ indica recibir menos de 1.000 euros mensuales, el $53.9 \%$ cobra entre 1.000 y 2.000 euros al mes, un $17.4 \%$ percibe entre 2.000 y 3.000 euros mensuales y el 2,6\% manifiesta ganar más de 3.000 euros al mes.

En el estudio actual, a la hora de analizar las variables de salud mental, se consideró impor tante tener en cuenta la identidad de género de los sujetos participantes en el mismo. Por ello, se procedió a categorizar a los sujetos en 4 grupos siguiendo la taxonomía, Bem (1974), i.e., masculino, femenino, andrógino e indiferenciado.

Así, las medias de las dimensiones de masculinidad-instrumentalidad $(M=63.09)$ y de feminidad-expresividad ( $M=68.40)$ sirvieron como punto de corte para establecer las cuatro categorías de identidad de género.Así, el grupo con identidad de género masculino lo fomaron aquellos sujetos con puntuaciones iguales o superiores a la media en la dimensión de masculinidad-instrumentalidad e inferiores a la media en la dimensión de feminidad-expresividad; el grupo con identidad de género femenina lo formaron aquellos sujetos con puntuaciones iguales o superiores a la media en la dimensión de feminidad-expresividad e inferiores a la media en la dimensión de masculinidad-instnumentalidad; el grupo de andróginos estuvo constituido por aquellos sujetos con puntuaciones iguales o superiores a las media de ambas dimensiones; $y$, finalmente, el grupo de indiferenciados se constituyó por aquellos sujetos con puntuaciones inferiores a las medianas en ambas dimensiones.

\section{Instrumentos}

Con el fin de establecer un perfil descriptivo de la muestra LGB que ha participado en el estudio, las personas respondieron a una serie de cuestiones sociodemográficas relativas a su sexo, edad, orientación sexual, estado civil, número de hijos/as, pareja actual y si reside con ella, localidad y provincia de residencia actual, ni vel de estudios, ocupación, religión, práctica religiosa, ideología política e ingresos mensuales.

Olweus Bully/Victim Questionnaire (Olweus, 1993). Instrumento de autoinforme que consta de 12 items, los cuales e valúan el nivel de bullying percibido según un formato de respuestas dicotómico («sí» vs. «no»). Ha sido utilizado de varias formas en otros estudios llevados a cabo en UK, Norte América, España, Portugal, Japón e Irlanda (ver Olweus, 1994; Smith y Shar p, 1994). Se utilizó la versión adaptada que Rivers (2000) utilizó para medir el acoso sufrido en la enseñanza secundaria (edades comprendidas entre 12 y 18 años). Se utilizaron dos indicadores de esta variable, el primero que hemos denominado ifuiste acosado?, recoge el hecho de haber sido o no víctima de b ullying durante la infancia y/o adolescencia. El segundo, más específico, centrado en el acoso por insultos relacionados con la orientación af ectivo-sexual indica si el sujeto ha sido o no víctima de b ullying por insultos relacionados con la orientación afectivo-sexual. El coeficiente alfa del cuestionario fue de 0.73 en el presente estudio.

Bem Sex-Role Inventory (Bem, 1974). Aplicamos la adaptación a la pob lación española llevada a cabo por Fernández (1983) y Sebastián, Frías y Ayuso (1990). Se trata de un instrumento de autoinforme que evalúa la identidad de género a través de dos dimensiones independien- 
tes, la instrumentalidad-masculinidad y la expresividad-feminidad. A través de este cuestionario, el sujeto indica cuál es el g rado de autodescripción en cada atributo que se presenta teniendo como referencia una escala tipo Likert de 1 («nunca») al 5 («siempre»). El inventario está compuesto por 60 atributos, de los cuales 20 representan la dimensión de expresividad o feminidad, 20 la de instrumentalidad o masculinidad, y los 20 restantes actúan como filtros y sirven como escala de deseabilidad social neutra respecto al sexo. Esta escala se utilizó en el desar rollo del inventario de Bem para cerciorarse de que el instr umento no estuviera registrando un sesgo de aquiescencia, es decir, una tendencia general a confirmar los rasgos socialmente deseados. El inventario ha sido uno de los instrumentos más utilizados en psicología, debido a que posibilita clasificar a los individuos como masculino, femenino y andrógino, y a las buenas propiedades psicométricas que presenta (índices alfa de Cronbach superiores a 0.80) (Holt y Ellis, 1998). Los coeficientes alfa obtenidos en este estudio fueron de 0,80 para la escala de masculinidad-instrumentalidad y de 0.81 para la de feminidad-expresividad.

Beck Depression Inventory (BDI; Beck, Ward, Mendelson, Mock y Erbaugh, 1961). Instrumento de autoinforme que permite cuantificar los síntomas depresi vos en poblaciones normales así como en poblaciones clínicas. No es un instrumento de diagnóstico sino que evalúa la severidad de los síntomas co gnitivos, afectivos, conductuales y psicofisiológicos de la depresión a través de una puntuación que se considera como una medida de la intensidad de la misma. Por lo tanto es una escala de estado de ánimo. La escala es una medida consistente, estable y válida (Beck, Steer y Garbin, 1988) y presenta buenas propiedades psicométricas (alfa de Cronbach de 0.83). En el estudio actual, se utilizó una versión reducida compuesta por 13 ítems (Beck et al., 1961) previamente adaptada y validada en nuestro conte xto (Páez, 1986), obteniéndose un índice de consistencia interna (alfa) en nuestra muestra de 0.88 .

Inventario de Situaciones de Respuestas de Ansiedad (ISRA; Miguel Tobal y Cano Vindel, 1986). Se utilizó una v ersión reducida para evaluar el nivel de ansiedad, la cual consta de
17 ítems con for mato de respuesta de autoinforme tipo Likert (Martínez-Sánchez et al., 1995). Es un instr umento de evaluación del nivel general de ansiedad que se obtiene como suma de las respuestas co gnitivas, fisiológicas y motoras. En el estudio actual, el v alor del coeficiente alfa obtenido ha sido de 0.93 .

Positive Affect-Negative Affect Schedule (PANAS; Watson, Clark y Tellegen, 1988). Utilizamos la versión española validada por Sandín et al. (1999). Evalúa dos dimensiones del afecto (afecto positivo y afecto ne gativo), cada una constituida por 10 ítems. Los ítems se contestan según una escala tipo Lik ert, con un rango de variación de 1 («nada») a 5 («mucho»). Los índices de consistencia inter na (alfa de Cronbach) son de 0.88 y de 0.89 para el afecto negativo y positivo respectivamente. Mide aspectos de equilibrio (balance) afectivo, tanto a nivel de estado como de rasgo. La diferencia entre las dos subescalas de afectividad positiva y negativa proporciona una medida del equilibrio afectivo del sujeto. En el estudio actual se le denominó a este resultado afectividad. En el presente estudio obtuvimos coeficientes alfa 0.81 y 0.92 para las escalas de afecto positi vo y afecto negativo, respectivamente.

Self-esteem Scale (SES; Rosenberg (1965). Se trata de una escala unidimensional, basada en el modelo de Guttman (1944), for mada por 10 ítems, de los cuales 5 se enuncian de forma positiva y 5 de forma negativa para controlar el efecto de aquiescencia. A través de la escala se obtienen datos sobre el sentimiento de satisfacción o insatisfacción que tiene la persona consigo misma. La fiabilidad interna de la escala original fue de 0.85 (alfa de Cronbach). En lo que respecta a la adaptación española de la escala, ha sido validada tanto en población adolescente (Atienza, Moreno y Balaguer, 2000) como en pob lación universitaria (Martín-Albo, Núñez, Navarro y Grijalvo, 2007). En el estudio actual, el valor del coeficiente alfa obtenido ha sido de 0.85 .

\section{Procedimiento}

Se utilizó un diseño selecti vo transversal. Dada la naturaleza de la pob lación objeto de estudio, la selección muestral se realizó median- 
te un muestreo no probabilístico. Se contactó con las principales asociaciones de gais, lesbianas y bisexuales de Guipúzcoa, Vizcaya y Navarra, concertándose una cita con la persona responsable con el fin de presentar el estudio y pedir su colaboración. Posteriormente, a cada miembro de la asociación se le en vió un sobre que incluía un cuestionario, una carta explicativa del estudio en el cual iba a par ticipar y otro sobre franqueado para reenviar el cuestionario una vez completado y así asegurar el anonimato de sus respuestas. Fueron distrib uidos un total de 400 cuestionarios de los cuales se recogieron 119. Los sujetos participaron anónimamente en el estudio y no recibieron compensación económica alguna por participar en el mismo.

\section{RESULTADOS}

\section{Descripción del tipo de acoso}

El $51.3 \%$ de los sujetos que componen la muestra indicaron haber sufrido algún tipo de acoso en su etapa escolar. De éstos, el $42.62 \%$ fue acosado de vez en cuando, un 1.64\% sufrió un acoso moderado y el $18.03 \%$ indicó haber sido víctima de acoso varias veces a la semana.
Por su parte, el $57.38 \%$ de estos sufrió acoso por motivo de su orientación afectivo-sexual.

En lo que respecta al modo en que han sido acosados, un 55.74\% de los sujetos indicaron haber sido objeto de insultos y un $34.43 \%$ de rumores. El 29.52\% de las personas indicaron haber sufrido otros tipos de acoso psicológico (aislamiento, intimidación, pintadas, etc.) y el 1. $64 \%$ indicó haber sido víctima de acoso físico (golpes o patadas)

En relación a la época en la que sufrieron el acoso, el $54.7 \%$ indica que éste tuю lugar mientras cursaban sus estudios primarios, el $23.4 \%$ lo sufrió en el bachiller/formación profesional, un $18.7 \%$ comenzó a sufrir acoso durante sus estudios primarios y éste se manturo durante el bachiller-formación profesional y sólo un 3.1\% indica haber sido acosado en la universidad.

Entre dichas causas destacan el peso, tamaño o forma del cuerpo (29.50\%), la apariencia o forma de vestir $(9.84 \%)$, debido a los amigos que tenían $(8.20 \%)$, por que no eran buenos haciendo deporte $(32.79 \%)$ y otras causas (21.31\%) (véase la Tabla 1).

Finalmente, cabe destacar que de quienes sufrieron un acoso severo $(18.3 \%)$ la mayoría de estos $(81.82 \%)$ indican que el motivo fue su orientación afectivo sexual.

Tabla 1. Frecuencias y porcentajes de las causas de acoso

\begin{tabular}{lrr}
\hline Modo de acoso & n acosados & $\%$ acosados \\
\hline Por peso/tamaño/forma & 18 & $29.50 \%$ \\
Por apariencia & 6 & $9.84 \%$ \\
Por insultos relacionados con la orientación afectivo-sexual & 35 & $57.38 \%$ \\
Por buen trabajo escolar & 10 & $16.39 \%$ \\
Por mal trabajo escolar & 3 & $4.92 \%$ \\
Por incapacidad física & 3 & $4.92 \%$ \\
Por los amigos & 5 & $8.20 \%$ \\
Por ser buen deportista & 3 & $4.92 \%$ \\
Por ser mal deportista & 20 & $32.79 \%$ \\
Por la ropa & 5 & $8.20 \%$ \\
Por otras causas & 13 & $21.31 \%$ \\
\hline
\end{tabular}

Acoso relacionado con la orientación afectivo-sexual y los datos sociodemográficos de la muestra

Los resultados obtenidos muestran que el acoso por insultos relacionado con la orienta- ción afectivo-sexual se relaciona con el sexo de los sujetos observados $(p=0.00)$, siendo los varones quienes más acoso han sufrido por esta razón con respecto a las mujeres, y con el tener pareja. Aquellas personas que fueron acosadas por insultos relacionados con su orientación 
afectivo-sexual tienen menos parejas en la actualidad que aquellos participantes que no fueron acosados $\left(\chi^{2}(1)=3.12 ; p=0.08\right)$. Asimismo, hay que destacar que aquellos participantes que viven en el contexto urbano han sufrido más acoso por su orientación afecti vo-sexual que aquellos que viven en el contexto rural aunque la prueba chi-cuadrado $\left(\chi^{2}(1)=1.97 ; p=0.16\right)$ mostró que las diferencias no son estadísticamente significativas. Por otro lado, no se encuentran diferencias estadísticamente significativas con el resto de las variables: estado civil, reside con su pareja, lugar de residencia, nivel de estudios, si es católico o si no lo es, g rado de práctica religiosa y con el grado de simpatía política (véase la Tabla 2).

Tabla 2. Características sociodemográficas de los acosados por insultos relacionados con la orientación afectivo-sexual y los no acosados

\begin{tabular}{|c|c|c|c|c|c|c|}
\hline & & \multicolumn{3}{|c|}{ AIOAS } & \multirow{2}{*}{$\chi^{2}$} & \multirow{2}{*}{$p$} \\
\hline & & Sí acosados & No acosados & Total & & \\
\hline \multirow{2}{*}{ Sexo } & Varón & 31 & 54 & 85 & \multirow[t]{2}{*}{8.27} & \multirow[t]{2}{*}{0.00} \\
\hline & Mujer & 3 & 29 & 32 & & \\
\hline \multirow[t]{2}{*}{ Orientación afectivo-sexual } & Homosexual & 34 & 80 & 114 & \multirow[t]{2}{*}{0.04} & \multirow[t]{2}{*}{0.84} \\
\hline & Bisexual & 1 & 3 & 4 & & \\
\hline \multirow[t]{5}{*}{ Estado civil } & Soltero/a & 28 & 62 & 90 & \multirow[t]{5}{*}{2.46} & \multirow[t]{5}{*}{0.65} \\
\hline & Vive en pareja & 1 & 3 & 4 & & \\
\hline & Pareja de hecho & 6 & 11 & 17 & & \\
\hline & Separado & 0 & 2 & 2 & & \\
\hline & Divorciado & 0 & 3 & 3 & & \\
\hline \multirow[t]{2}{*}{ Tiene actualmente pareja } & Sí & 15 & 52 & 67 & \multirow[t]{2}{*}{3.12} & \multirow[t]{2}{*}{0.08} \\
\hline & No & 19 & 32 & 51 & & \\
\hline \multirow[t]{2}{*}{ Reside con su pareja } & Sí & 9 & 32 & 41 & \multirow[t]{2}{*}{2.00} & \multirow[t]{2}{*}{0.16} \\
\hline & No & 23 & 43 & 66 & & \\
\hline \multirow[t]{2}{*}{ Contexto urbano-rural } & Urbano & 19 & 57 & 76 & \multirow[t]{2}{*}{2.17} & \multirow[t]{2}{*}{0.16} \\
\hline & Rural & 16 & 27 & 43 & & \\
\hline \multirow[t]{2}{*}{ Nivel de estudios } & Superiores & 17 & 39 & 56 & \multirow[t]{2}{*}{1.84} & \multirow[t]{2}{*}{0.83} \\
\hline & No Superiores & 18 & 45 & 63 & & \\
\hline \multirow[t]{2}{*}{ Católico/a } & Sí & 14 & 34 & 48 & \multirow[t]{2}{*}{0.01} & \multirow[t]{2}{*}{0.96} \\
\hline & No & 19 & 47 & 66 & & \\
\hline \multirow[t]{2}{*}{ Grado de práctica religiosa } & Nunca & 24 & 52 & 76 & \multirow[t]{2}{*}{1.73} & \multirow[t]{2}{*}{0.49} \\
\hline & Alguna vez & 11 & 32 & 43 & & \\
\hline \multirow[t]{2}{*}{ Grado de simpatía política } & Izquierdas & 27 & 72 & 99 & \multirow[t]{2}{*}{1.41} & \multirow[t]{2}{*}{0.25} \\
\hline & Derechas & 8 & 12 & 20 & & \\
\hline
\end{tabular}

Nota: AIOAS: acoso por insultos relacionados con la orientación afectivo-sexual.

\section{Relación entre las variables de salud mental}

Con el fin de analizar las relaciones existentes entre las variables de salud mental se procedió a realizar un análisis de cor relaciones bivariadas. Los resultados indican que aquellos sujetos con mayor autoestima manifiestan menores niveles de ansiedad y depresión $(r=0.59$, $r=0.67$ respectivamente) al mismo tiempo que indican un mejor estado emocional o balanza de afectos. La ansiedad por su parte se vincula con mayores niveles de depresión $(r=0.72)$ y una menor autoestima y peor balanza de afectos $(r=0.69)$. A su vez, los datos muestran que la ansiedad se relaciona de for ma directa con la depresión y de forma inversa con la autoestima y el estado emocional o la balanza de afectos siendo estas relaciones más intensas en el caso de aquellos sujetos que fueron acosados. 
Comparación de resultados en las variables de salud mental entre los sujetos acosados y no acosados

Con el objeto de comparar los resultados de las variables de la salud mental entre aquellos participantes que han sido acosados por insultos relacionados con la orientación afectivo-sexual y los que no, se procedió a analizar los datos mediante la prueba $t$ de Student. Las medias obtenidas apuntan a que aquellos sujetos que han sufrido acoso tienen ma yores niveles de ansiedad, depresión, un peor equilibrio afectivo y una autoestima menor si bien estos resultados no son estadísticamente significativos (véase la Tabla 3).

Tabla 3. Diferencias en las variables de salud mental de los acosados por insultos relacionados con la orientación afectivo-sexual

\begin{tabular}{lccccccc}
\hline & AIOAS & $n$ & Media & DT & $t$ & g.l. & $p$ \\
\hline \multirow{2}{*}{ Autoestima } & No & 84 & 40.03 & 6.04 & & & 0.17 \\
& $\mathrm{Si}$ & 35 & 38.22 & 7.65 & 1.25 & 118 & \\
Ansiedad & $\mathrm{No}$ & 81 & 31.06 & 9.74 & & & \\
& $\mathrm{Si}$ & 35 & 35.74 & 13.41 & -1.87 & 115 & 0.07 \\
Depresión & $\mathrm{No}$ & 79 & 16.35 & 4.25 & & & 0.13 \\
& $\mathrm{Si}$ & 34 & 17.77 & 4.55 & -1.60 & 112 & \\
Afectividad & $\mathrm{No}$ & 81 & 11.42 & 10.72 & & & 0.24 \\
& $\mathrm{Si}$ & 34 & 8.94 & 9.33 & 1.12 & 114 & \\
\hline
\end{tabular}

Comparación de las variables de salud mental en función del género

Se clasificó a los sujetos en cuatro categorías de identidad género: masculino, femenino, andrógino e indiferenciado. Para examinar la variación de las puntuaciones en salud mental en función de las cuatro categorías de identidad de género se aplicó un diseño de ANOVA de un factor (factor de grupos, según las 4 categorías de identidad de género) para cada una de las variables de salud mental (variables dependientes).

Como indicamos en la Tabla 4, los resultados indican efectos significativos de las categorías de identidad de género para cada una de las cuatro variables dependientes $(p<.01)$. No obstante, al comparar entre los diferentes $\mathrm{g}$ rupos (categorías de identidad de género), únicamente encontramos diferencias significativas entre los grupos femenino y andrógino en autoestima y afectividad, y entre los g rupos andrógino e indiferenciado en depresión. En comparación con el g rupo femenino, el g rupo andrógino exhibe puntuaciones significativamente mayores en autoestima y afecti vidad (mayor predominancia del afecto positivo). Así
Tabla 4. Puntuaciones en las variables de la salud mental en función del género

\begin{tabular}{|c|c|c|c|c|c|}
\hline & & $n$ & Media & $D T$ & $F$ \\
\hline \multirow[t]{5}{*}{ Autoestima } & MAS & 18 & 42.01 & 5.02 & $4.42^{* *}$ \\
\hline & FEM & 28 & $37.82^{\mathrm{a}}$ & 6.92 & \\
\hline & AND & 30 & $42.59^{b}$ & 4.50 & \\
\hline & IND & 18 & 38.78 & 5.77 & \\
\hline & Total & 94 & 40.33 & 5.95 & \\
\hline \multirow[t]{5}{*}{ Ansiedad } & MAS & 17 & 27.42 & 6.82 & $3.80^{* *}$ \\
\hline & FEM & 27 & 34.93 & 10.97 & \\
\hline & AND & 30 & 28.40 & 7.51 & \\
\hline & IND & 18 & 34.55 & 12.42 & \\
\hline & Total & 92 & 31.34 & 10.04 & \\
\hline \multirow[t]{5}{*}{ Depresión } & MAS & 16 & 15.00 & 3.14 & $3.87^{* *}$ \\
\hline & FEM & 26 & 17.30 & 4.95 & \\
\hline & AND & 30 & $14.65^{\mathrm{a}}$ & 2.12 & \\
\hline & IND & 17 & $17.84^{b}$ & 4.74 & \\
\hline & Total & 89 & 16.10 & 4.02 & \\
\hline \multirow[t]{5}{*}{ Afectividad } & MAS & 17 & 15.34 & 8.28 & $3.96^{* *}$ \\
\hline & FEM & 28 & $7.97^{\mathrm{a}}$ & 10.06 & \\
\hline & AND & 29 & $15.32^{\mathrm{b}}$ & 9.70 & \\
\hline & IND & 18 & 9.80 & 8.79 & \\
\hline & Total & 92 & 12.01 & 9.85 & \\
\hline
\end{tabular}

Nota: MAS: masculino; FEM: femenino; AND: andrógino; IND: indiferenciado.

Los superíndices ${ }^{\mathrm{a}, \mathrm{b}}$ indican diferencias entre grupos $(p<.01$; prueba post-hoc de Boferroni).

$* * p<0.01$. 
mismo, encontramos niveles significativamente mayores en depresión en el grupo indiferenciado que en el g rupo andrógino. Como puede apreciarse en la tabla, no encontramos diferencias significativas referidas al grupo masculino, aunque en general las puntuaciones de este grupo parecían bastante similares al grupo andrógino.

Interacción entre la identidad de género y el acoso en relación con las variables de salud mental

Con el fin de examinar la existencia de una posible interacción entre las categorías de identidad de género y el acoso relacionado con la orientación afectivo-sexual, respecto a su posible efecto sobre la salud mental, aplicamos un diseño de ANOVA de 4 (categorías de identidad de género) $\times 2$ (acoso vs. no acoso) para cada una de las cuatro variables dependientes (autoestima, ansiedad, depresión y afectividad). La interacción entre ambos factores no resultó ser estadísticamente significativa para ninguna de las variables de salud mental. No obstante, tal y como se indican en la Figura 1, los datos indican cierta tendencia en el sentido de que aquellos sujetos con identidades de género masculinas y andróginas parecen tener mayores niveles de autoestima y una afectividad más positiva y menores niveles de ansiedad y depresión que aquellos sujetos con identidades de género femeninas e indiferenciadas. Se puede apreciar la tendencia a la interacción entre la $\mathrm{v}$ ariable de identidad de género y de acoso por insultos relacionados con la orientación afectivo-sexual. A pesar de que la interacción existente entre los distintos grupos y la existencia o no del acoso no es significativa, se pueden apreciar diferencias muy marcadas que indican tendencias que revelan un comportamiento diferencial de cada uno de los grupos. El bajo tamaño muestral puede ser una razón de la no significatividad de la interacción. A pesar de ello, se considera importante revelar la existencia de esta tendencia que consideramos debe ser objeto de análisis posterior con un incremento del número de participantes. A su vez, destacar que el acoso disminuye los niveles de ansiedad en las perso- nas clasificadas en las cate gorías masculina y femenina y los niveles de depresión en los clasificados en la cate goría femenina (véase la Figura 1).

\section{DISCUSIÓN}

Los resultados del estudio indican una prevalencia del acoso entre los sujetos LGB, fundamentalmente con relación al acoso por motivo de orientación afectivo-sexual y que tales situaciones de victimización sufridas tiene consecuencias negativas en la salud mental de la persona. Como cabría esperar, el porcentaje de acoso es ligeramente superior al 34\% obtenido recientemente por Magaz et al. (2011). A este respecto, los sujetos que sufrieron experiencias de victimización en el contexto escolar/educativo por motivo de orientación afectivo-sexual son los que presentan ma yores niveles de depresión y ansiedad, menores niveles de autoestima y un peor balance (equilibrio) de afectos. Estos resultados apoyan evidencias empíricas que apuntan que el estatus de víctima parece ser relativamente estable durante el tiempo (Olweus, 1993; Rivers, 2004; Rivers y Cowie, 2006) y que como consecuencia de recordar las experiencias de acoso sufridas en la escuela se mantienen los trastornos psicológicos (Rivers y Cowie, 2006). Es preciso indicar, en base al limitado número de participantes del estudio, que, aunque las diferencias no son significativas, el tamaño de las mismas indican que las tendencias son evidentes y que necesitan ser objeto de estudio con muestras más impor tantes.

A su vez, los resultados confirman resultados similares donde se encontraron síntomas de estrés postraumático en un $17 \%$ de los sujetos que sufrieron bullying debido a su actual o per cibida orientación sexual (Rivers, 2004). Además, estos sujetos tuvieron más probabilidades de sufrir depresión que el resto de los par ticipantes.

Por su parte la identidad de género ha resultado relevante como factor explicativo del bienestar psicológico. Como muestran los resultados, no sólo la alta instr umentalidad (Sharpe, Heppner y Dixon, 1995) sino que ésta combi- 


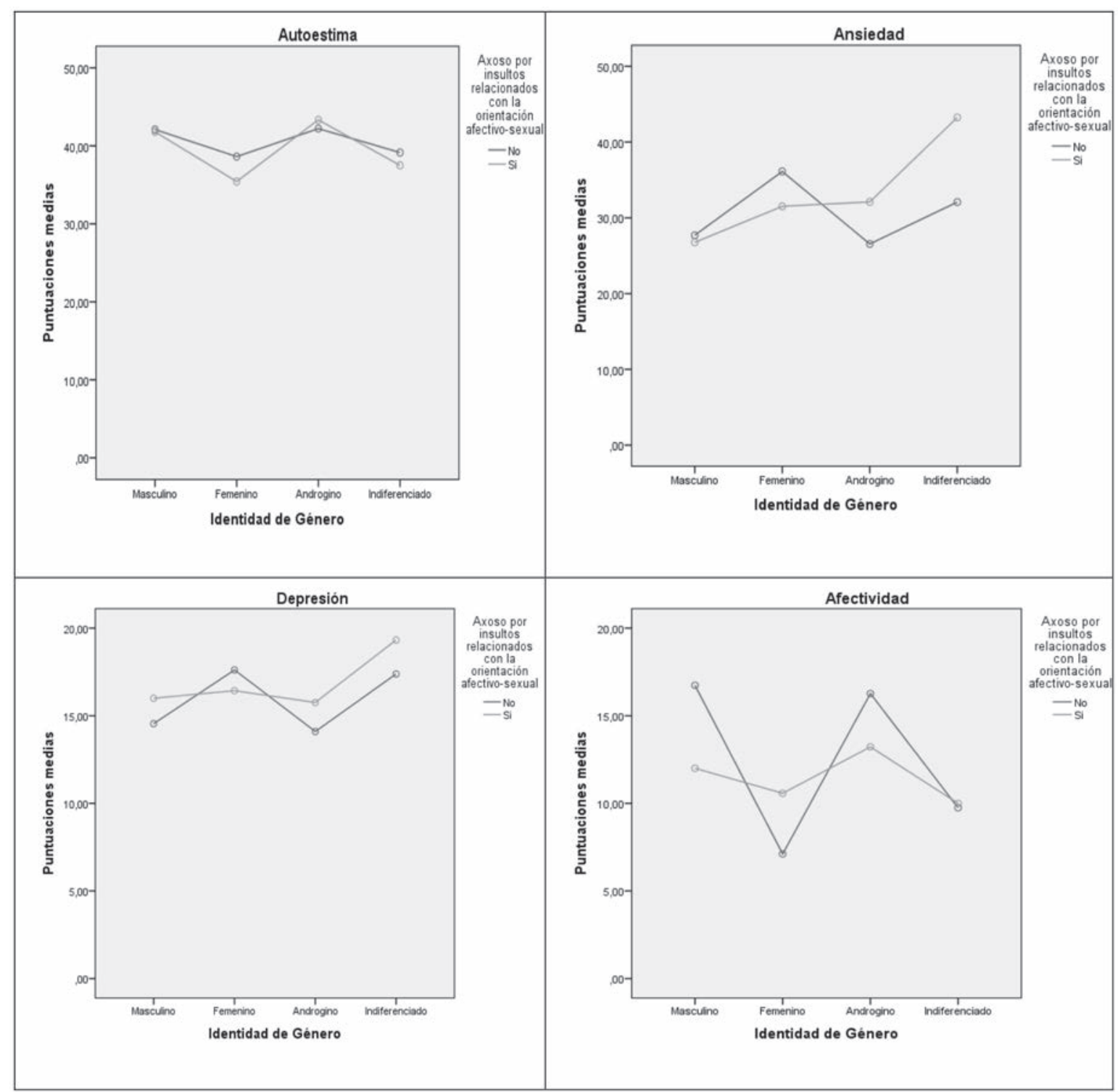

Figura 1. Interacción de la identidad de género y el acoso por insultos relacionados con la orientación afecti vo-sexual referida a las variables de autoestima, ansiedad, depresión y afectividad (ver texto).

nada con la baja expresividad (Wagner y Compas, 1990), se asocia al bienestar psicológico. Concretamente los resultados muestran que la baja masculinidad-instrumentalidad está relacionada con una baja autoestima (Payne, 1987; Sharpe, Heppner y Dixon, 1995), ansiedad (Payne, 1987), y depresión y una pobre adaptación (Bromberger y Matthews, 1996; Whitley, 1984). Asimismo, la alta masculinidad-instrumentalidad se asocia positivamente de forma más fuerte con la autoestima (Whitley, 1984) así como con niveles más bajos de depresión (Bar ret y White, 2002; Carlson y Baxter, 1984). Así, los resultados obtenidos en este estudio indican que el bienestar psicológico se explicaría más adecuadamente a partir del modelo de masculinidad-instrumentalidad que a partir del modelo de la androginia apoyando resultados empíricos que muestran el efecto moderador de la instrumentalidad en la relación de sucesos estresantes negativos y síntomas psicológicos (Bar ret y White, 2002; Bromberger y Matthews, 1996). 
A modo de conclusión, los resultados del estudio parecen indicar, que los procesos de estrés que sufren las personas LGB por per tenecer a una categoría social estigmatizada relacionada con la sexualidad influyen en la salud mental de estos sujetos apoyando el modelo de estrés de minorías que plantea Me yer (1995, 2003). A este respecto, aquellos sujetos que han sufrido experiencias de victimización por motivo de orientación afectivo-sexual en el pasado indican niveles superiores de depresión y ansiedad y peores niveles de autoestima y equilibrio afectivo.

El estudio posee algunas limitaciones que deseamos poner de manifiesto. En primer lugar, a día de hoy, no está consensuado lo que es ser homosexual ni sus indicadores, lo cual dificulta elaborar una clasificación de la homosexualidad por la comunidad científica. La forma en que se ha evaluado a lo largo de los años los estudios llevados a cabo con personas LG para considerar si son homosexuales carece de cualquier rigor en muchos de los casos (BaileAyensa, 2008) y es debido a ello que en futuras investigaciones se deberá ahondar en el tratamiento del constructo de la orientación afectivo-sexual. A su vez, en lo que respecta al sistema de muestreo, el estudio actual utiliza una muestra accesible que no es generalizable en los resultados a la población. Así, es fundamental que los estudios venideros sean estudios aleatorios que puedan ser generalizab les. Asimismo, sería conveniente disponer de datos de sujetos heterosexuales que sirvieran como grupo de comparación y per mitiera, además, el estudio de la controversia existente en los resultados obtenidos en diferentes in vestigaciones acerca de las diferencias en varones y mujeres homosexuales y heterosexuales y bisexuales. Además, el desequilibrio existente entre el número de varones y de mujeres que ha participado compromete los resultados. A este respecto, se considera prioritario aumentar el número de mujeres en el estudio para la realización de posteriores análisis. Para concluir, una importante limitación se refiere a la naturaleza de los sujetos que componen el estudio. El hecho de ser sujetos homosexuales que participan activamente en colectivos de gais, lesbianas, bisexuales y transexuales, hacen que sean personas que comparten un mismo discurso social y, en nuestra opinión, pueden ser sujetos no representativos del resto de personas LGB. Sin embargo, se trata de un problema de difícil solución dada la dificultad o imposibilidad de acceder a estos sujetos. A este respecto, una limitación adicional, incluye el sesgo que se introduce en las medidas de auto-informe. Al ser todas las medidas de auto-informe, las respuestas dependen de la precisión de los participantes y a menudo comparten un sesgo común en las respuestas.

\section{REFERENCIAS}

Atienza, L. F., Moreno, Y., y Balaguer, I. (2000). Análisis de la dimensionalidad de la escala de autoestima de Rosenberg en una muestra de adolescentes v alencianos. Revista de Psicología Universitas Tarraconensis, 1, 29-42.

Baile Ayensa, J. I. (2008). Estudiando la homosexualidad. Teoría e investigación. Madrid: Ediciones Pirámide.

Barret, A., \& White, H. (2002). Trajectories of gender role orientations in adolescence and early adulthood: A prospective study of the mental health ef fects of masculinity and femininity. Journal of Health and Social Behavior, 43, 451- 468.

Beck, A. T., Steer R. A., \& Garbin, M. C. (1988). Psychometric properties of the Beck Depression Inventory: Twenty-five years of evaluation. Clinical Psychology Review, 8, 77-100.

Beck, A.T., Ward, C. H., Mendelson, M., Mock, J ., \& Erbaugh, J. (1961). An inventory for measuring depression. Archives of General Psychiatry 4, 561-571.

Bem, S. L. (1974). The measurement of psychological androgyny. Journal of Consulting and Clinical Psychology, 42, 155-162.

Birkett, M., Espelage, D.L., \& Koenig, B. (2009). LGB and questioning students in schools: The moderating effects of homophobic bullying and school climate on negative outcomes. Journal of Youth and Adolescence, 38, $989-1000$.

Blashill, A. J., \& Hughes, H.M. (2009). Gender role and gender role conflict: Implications for counseling with gay men. Journal of Gay and Lesbian Mental Health, 13, 170-186.

Bontempo, D., \& D’Augelli, A. (2002). Effects of atschool victimization and sexual orientation on lesbian, gay, or bisexual youths' health risk behaviors. Journal of Adolescent Health, 30, 364-74.

Bromberger, J.T., \& Matthews, K.A. (1996). A «feminine» model of vulnerability to depressi ve symptoms: a longitudinal investigation of middle-aged women. Journal of Personality and Social Psychology, 70, 591-598. 
Carlson, H. M. y Baxter, L. A. (1984). Androgyny, depression, and self-esteem in Irish homose xual and heterosexual males. Sex Roles, 10, 457-467.

Eccles, J. S., \& Bryan, J. (1994). Adolescence: Critical crossroad in the path of gender -role development. In M. Stevenson (Ed.), Gender roles through the life span (pp. 111-148). Muncie, IN: Ball State University.

Espelage, D. L., Aragon, S. R., \& Birk ertt, M. (2008). Homophobic teasing, psychological outcomes, and sexual orientation among high school students. What influence do parents and schools ha ve? In D.L. espelage, \& S.M. Swearer (Eds.), Sexual Orientation, Homophobia, Bullying, and Psychological Adjustment During Adolescense [Special issue]. School Psychology Review, 37, 202-216.

FELGTB (2012). Acoso escolar homofóbico y riesgo de suicidio en adolescentes y jóvenes $L G B$. Recuperado el 14 de Marzo de 2013, de: http://www .felgtb.org/ rs/1584/d112d6ad-54ec-438b-93584483f9e98868/91c/filename.

Fernández, J. (1983). Nuevas perspectivas en la medida de la masculinidad y feminidad. Madrid: Editorial de la Universidad Complutense.

Ferreira, E., Granero, R., Noorian, Z., Romero, K., y Doménech-Llaberia, E. (2012). Acontecimientos vitales y sintomatología depresiva en población adolescente. Revista de Psicopatología y Psicología Clínica, 17, 123-136.

Graber, J. A., \& Archibald, A. B. (2001). Psychosocial change at puberty and beyond: Understanding adolescent sexuality and sexual orientation. In A. R. D'Augelli \& C. J. Patterson (Eds.), Lesbian, gay, and bisexual identities and youth: psyc hological Perspectives (pp. 3-26). New York: Oxford University Press.

Guttman, L.A. (1944). A basis for scaling qualitative data. American Sociological Review, 9, 139-150.

Holt, C.L., \& Ellis, J.B. (1998). Assessing the current validity of the Bem Sex-Role Inventory. Sex Roles, 39, 929-941.

Kosciw, J. G., Greytak, E. A., Diaz, E. M., \& Bartkiewicz, M. J. (2010). The 2009 National Sc hool Climate Survey: The experiences of lesbian, gay, bisexual, and transgender youth in our nation's school. New York, NY: GLSEN.

Magaz, A. M., Chorot, P., Sandin, B., Santed, M. A. y Valiente, R. M. (2011). Estilos de apego y acoso entre iguales (bullying) en adolescentes. Revista de Psicopatología y Psicología Clínica, 16, 207-221.

Martín-Albo, L., Nuñez, J., Navarro, J., \& Grijalvo, F. (2007). The Rosenberg Self-Esteem Scale: Translation and validation in university students. Spanish Journal of Psychology, 10, 458- 468.

Martínez-Sánchez, F., Arias, A., Miguel-Tobal, J.J., CanoVindel, A., García Sevilla, J. y San Pedro. R.
(1995). Manual del Inventario de Situaciones y Respuestas de Ansiedad en el Aprendizaje de Vuelo. (I.S.R.A.-A.V.). Madrid: Ministerio de Defensa. Universidad de Murcia.

Meyer, I. H. (1995). Minority stress and mental health oer gay men. Journal of Health and Social Behavior, 36, 38-56.

Meyer, I. H. (2003). Prejudice, social stress, and mental health in lesbian, gay, and bisexual populations: Conceptual issues and research evidence. Psychological Bulletin, 129, 674-697.

Meyer, I. H. (2007). Prejudice and discrimination as social stressors. In I. H. Meyer and M. E. Northridge (Eds.), The health of sexual minorities: Pub lic health perspectives on lesbian, gay, bisexual and transgender populations (pp. 242-267). New York: Spring.

Miguel Tobal, J.J. y Cano Vindel, A. (1986). Manual del Inventario de Situaciones y Respuestas de Ansiedad -ISRA-. Madrid: TEA. (2 ${ }^{\mathrm{a}}$ Ed., 1988; $3^{\mathrm{a}}$ Ed., 1994).

Mireshghi., S.I., \& Matsumoto. D. (2008). Percieved cultural attitudes toward homosexuality and their effects on Iranian and American sexual minorities. Cultural Diversity and Ethnic Minority Psychology, 14, 372-376.

Olweus, D. (1993). Bullying at school: what we known and what we can do. Oxford: Blackwell.

Olweus, D. (1994). Annotation: Bullying at school: Basic facts and effects of a school based inter vention program. Journal of Child Psychology and Psychiatry, 35, 1171-1190.

Páez, D. (1986). Salud mental y factores psicosociales. Madrid: Fundamentos.

Patterson, C. J. (1995). Families of the lesbian baby boom: parents' division of labor and chilren's adjustment. Developmental Psychology, 31, 115-123.

Payne, F.D. (1987). «Masculinity», «femininity», and the complex construct of adjustment. Sex Roles, 17, 359-374.

Poteat, V. P., Aragon, S. R., Espelage, D. L., \& Koenig, B. W. (2009). Psychosocial concerns of sexual minority youth: Complexity and caution in group differences. Journal of Consulting and Clinical Psychology, 77, 196-201.

Quiceno, J. M., Mateus, J., Cardenas M., Villareal, D. y Vinaccia, S. (2013). Calidad de vida, resiliencia e ideación suicida en adolescentes víctimas de ab uso sexual. Revista de Psicopatología y Psicología Clínica, 18, 107-117.

Rivers I. (2000). Social exclusion, absenteeism, and sexual minority youth. Support for Learning, 5, 13-18.

Rivers, I. (2004). Recollections of bullying at school and their long-term implications for lesbians, gay, men, and bisexuals. Crisis, 25, 169-175.

Rivers, I., \& Cowie, H (2006) Bullying and homophobia at UK schools: A perspective on factors affecting resilience and recovery. Journal of Gay and Lesbian Issues in Education, 3, 11-43. 
Rivers, I., \& Noret, N. (2008). Well-being among samesex- and opposite-sex-attracted youth at school. School Psychology Review, 32, 174-187.

Rosenberg, M. (1985). Self-concept and psycholo gical well-being in adolescence. In R.L. Leak y (Ed.), The development of the self (pp. 205-246). New York: Academic Press.

Ryan, C., Huebner, D., Diaz, R. M., \& Sanchez, I (2009). Family rejection as a predictor of ne gative health outcomes in white and Latino lesbian, gay and bisexual young adults. Pediatrics, 123, 346-352.

Sandín, B., Chorot, P., Lostao, L., Joiner, T.E., Santed, M.A., y Valiente, R.M. (1999). Escala PANAS de afecto positivo y negativo: Validación factorial y convergencia transcultural. Psicothema, 11, 37-51.

Sebastián, J., Frías, J. I., y Ayuso, P. (1990). Análisis psicométrico y comparativo entre el Inventario del Rol Sexual (BSRI) y sus formas reducidas. Investigaciones Psicológicas, 8, 239-263.

Sharpe, M. J., Heppner P. P., \& Dixon W. A. (1995). Gender role conflict, instrumentality, expressiveness, and well being in adult men. Sex Roles, 33, 1-18.
Smith, P. K., \& Sharp, S. (1994). School bullying. Insights and perspectives. Londres: Routledge.

Swearer, S. M., Turner, R. K., Givens, J. E., \& Pollack, W. S. (2008). «You're so gay!»: Do different forms of bullying matter for adolescent males? School Psychology Review, 37, 160-173.

Takács, J. (2006). Social Exclusion of LGBT youth in Europe, ILGA-Europe and IGL YO publication. Available from www.ilga-europe.org/europe/ publications/non_periodical/.

Wagner, B. M., \& Compas, B . E. (1990). Gender, instrumentality and expressivity: Moderators of the relation between stress and psychological symptoms during adolescence. American Journal of Community Psychology, 18, 383-406.

Watson, D., \& Clark, L. A. (1984). Negative affectivity: The disposition to experience aversive emotional states. Psychological Bulletin, 96, 465-490.

Whitley, B.E. (1984). Sex-role orientation and psychological well-being: Two meta-analyses. Sex Roles, 12, 207-225. 